\title{
Initial TASAR Operations Onboard Alaska Airlines
}

\author{
David J. Wing* \\ Kelly A. Burke, Ph.D. ${ }^{\dagger}$ \\ Kathryn Ballard \\ NASA Langley Research Center, Hampton, VA 23681, USA \\ Jeffrey Henderson, Ph.D. $\$$ \\ SAIC, Andover, MA 01810, USA \\ Jared Woodward** \\ Alaska Airlines, Seattle, WA 98188, USA
}

\begin{abstract}
NASA and Alaska Airlines jointly conducted an operational evaluation of NASA's Traffic Aware Strategic Aircrew Request (TASAR) concept. Featuring a NASA-developed cockpit automation tool, the Traffic Aware Planner (TAP), and leveraging the emerging "connected aircraft" architecture, TASAR enables pilots to identify route changes compatible with nearby traffic and airspace constraints that improve flight efficiency and are more likely to receive Air Traffic Control approval. TASAR was developed in anticipation of saving fuel and flight time, thereby providing immediate and pervasive benefits to the aircraft operator. Following a previously reported, extensive program of integration and preliminary in-flight testing, the operational evaluation of TASAR in revenue service by Alaska Airlines took place from July 2018 to April 2019. This paper describes the operational evaluation, the methodology for quantifying operational benefits, preliminary benefit results, and other unexpected impacts of TASAR operations during revenue flights. Based on the subset of data available at publication, preliminary estimated benefits averaged about $\$ 92$ per flight across all flights including those without benefit. Identified factors indicate the estimated benefit per flight was likely a conservative estimate. Benefit categories including home runs, cumulative small gains, and plan validation benefits are illustrated with specific examples observed during the operational evaluation.
\end{abstract}

\section{Introduction}

he NASA concept of Traffic Aware Strategic Aircrew Requests (TASAR) took a significant step forward in 2018. Borne from NASA's research on advanced operational autonomy in the early 2000s, TASAR completed its transition from a research concept to an operational capability under evaluation by a prominent United States air carrier in revenue service. Working as partners under a NASA Space Act Agreement, NASA and Alaska Airlines (Alaska) equipped three Boeing 737-900ER aircraft with the NASA-developed TASAR cockpit automation tool, the Traffic Aware Planner (TAP). Using these aircraft, Alaska conducted an operational evaluation of TASAR on select revenue service flights from July 2018 to April 2019. TAP was approved by the Federal Aviation Administration (FAA) as an Electronic Flight Bag (EFB) software application so that it could be used operationally during the evaluation. Collaborating with NASA and Alaska to implement the TASAR system (hardware and software) on the three aircraft were commercial aviation industry leaders Collins Aerospace, Gogo Commercial Aviation, and Aviation Communications \& Surveillance Systems (ACSS).

\footnotetext{
${ }^{*}$ ATM Research Engineer, Crew Systems \& Aviation Operations, Mail Stop 152, AIAA Associate Fellow

${ }^{\dagger}$ Human Factors Research Scientist, Crew Systems \& Aviation Operations, Mail Stop 152, AIAA Member

* Statistical Engineer, Systems Engineering and Engineering Methods Branch, Mail Stop 290

$\S$ Senior Research Engineer, 2 Tech Dr, AIAA Senior Member

${ }^{* *}$ Technical Pilot, Alaska Airlines Flight Operations, 2651 S $1192^{\text {nd }}$ St.
} 


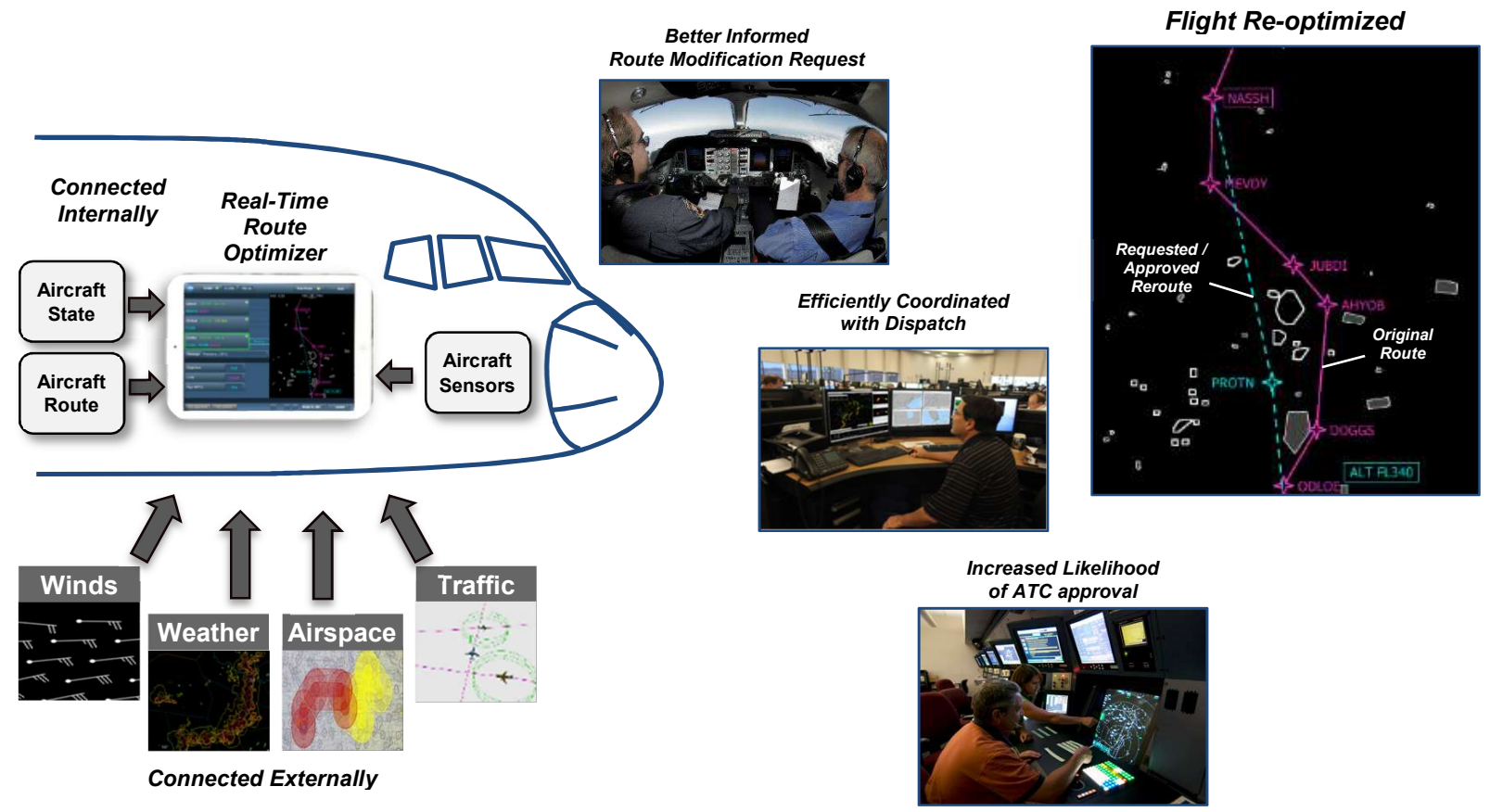

Figure 1. TASAR leverages data connectivity and a real-time route optimizer, TAP, to produce optimized flight benefits.

TASAR leverages the emerging revolution of the "connected aircraft" wherein access to operational information by systems onboard and off the aircraft becomes ubiquitous. [1] Technologies such as EFBs, aircraft interface devices (AID), and in-flight connectivity (IFC) solutions offer computing power and unprecedented Internet-Protocol-based connectivity to EFB software applications. Together, they are changing the landscape in which new operational capabilities are envisioned and implemented onboard aircraft. As illustrated in Figure 1, NASA's TASAR concept [2] features pilot use of a "connected EFB" application with a route optimization engine that consumes data retrieved from multiple sources:

1. onboard flight management system (FMS) (e.g., aircraft state and route data);

2. other onboard avionics systems (e.g., Automatic Dependent Surveillance-Broadcast (ADS-B) traffic data); and

3. ground-based data sources (e.g., wind forecasts, convective weather products, special use airspace (SUA) activation schedules).

Combined with pilot inputs via a graphical user interface, these data are processed by a search algorithm that identifies route optimization opportunities. The flight crew reviews these opportunities and, if appropriate, requests changes in the aircraft's lateral path and/or altitude (i.e. route modification) from air traffic control (ATC).

TAP is NASA's prototype of the TASAR route optimization EFB application. [3] Shown in Figure 2, TAP is a cockpit-based software tool for pilots to identify in-flight route-optimization opportunities that - by also avoiding nearby air traffic, weather, special-use airspace, and other constraints - are more likely to be approved by ATC when requested by the pilot. Leveraging its installation onboard the aircraft with direct access to real-time information about the aircraft's current state, route, and performance parameters, TAP monitors for potential route modifications (lateral path and/or altitude changes) that will improve a user-specified optimization objective, such as saving fuel or saving flight time. The pilot can dynamically update TAP's route optimization objective during a flight. Airlines are typically concerned with minimizing trip cost, which is a variable function of flight time and fuel costs. TAP supports any definition of fuel- and time-based optimization criteria, and its design enables future incorporation of other factors such as turbulence, i.e., ride quality. Using aircraft traffic information from the ADS-B In system, as well as information on airspace winds, weather, restricted airspace status, and other ATC constraints received by airborne internet or other data links, TAP incorporates these constraints into its computation of a set of route-optimization advisories for pilot review. TAP advisories are produced by a hybrid genetic/exhaustive search algorithm and include lateral-only, vertical-only, and combined lateral/vertical route modifications. Based on TAP recommendations, the pilot may make a route modification request to ATC using standard voice-request procedures. Because TAP-generated 


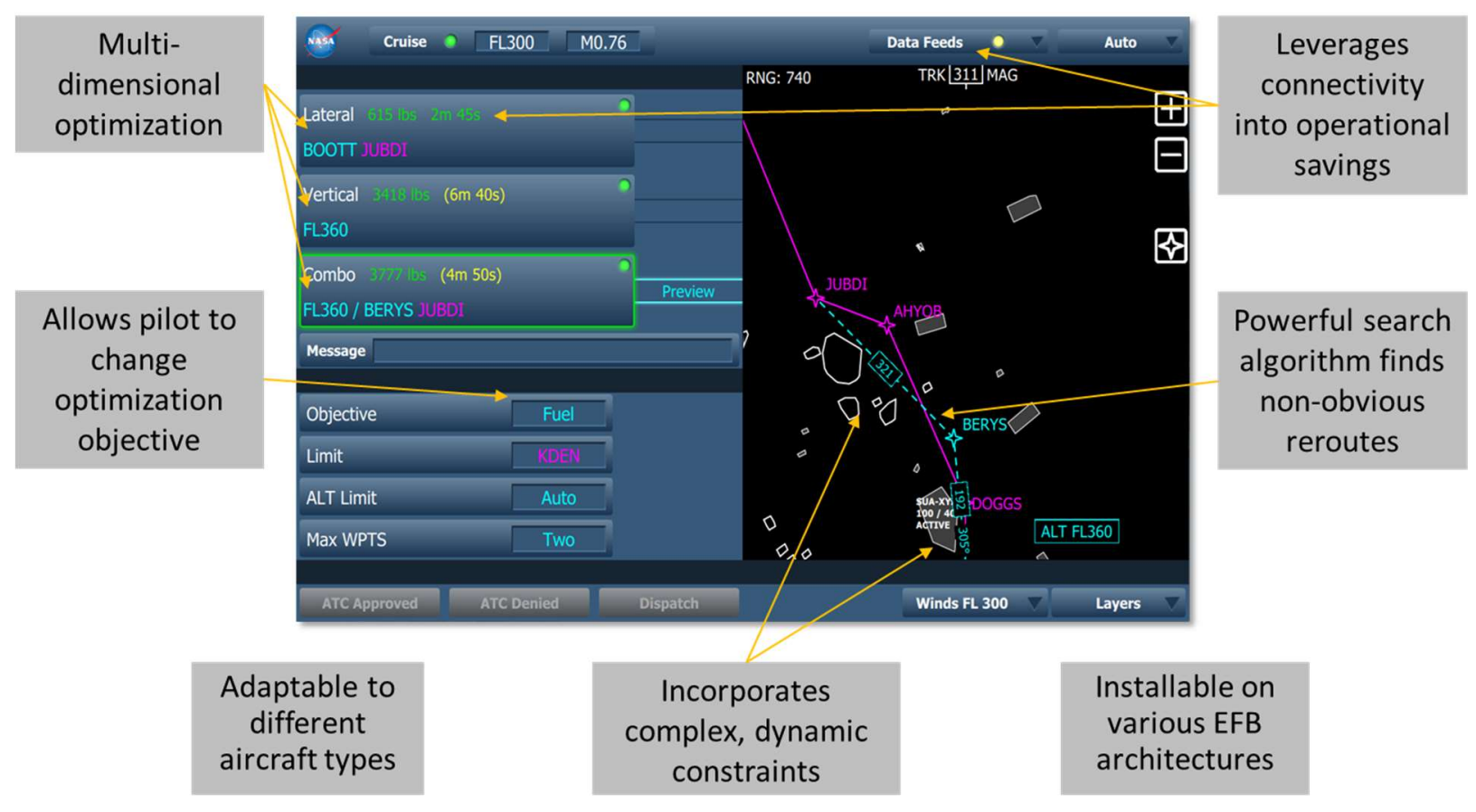

Figure 2. TAP is a NASA prototype interactive route-optimization application for pilots.

solutions incorporate traffic avoidance and other operational constraints, they are more likely to be approved by ATC and therefore more likely to result in actual achieved benefits by the aircraft operator. In addition to providing automated route-modification advisories, the TAP graphical user interface (i.e., TAP Display) also allows the pilot to enter a desired route change manually for TAP to analyze for outcomes and conflicts. TAP is NASA's state-of-the-art prototype EFB software application that achieves the TASAR concept and was awarded NASA Software of the Year in 2016.

Previous publications have described TASAR's research and development history [4] [5], benefits analyses [6] [7] [8], safety and certification analyses [9] [10], TAP design and functionality [3] [11], flight testing [12], and implementation at Alaska as a fully functioning EFB application approved by the FAA for operational use in revenue service [13]. This paper will describe the operational evaluation of TASAR by Alaska in revenue service operations. Section II will describe the approach used for conducting TASAR flight operations. Section III will describe the methodology developed for quantifying achieved benefits and present preliminary benefit findings. Section IV will describe several operational benefit categories of TASAR observed in flight. Section V will present conclusions.

\section{Approach for Conducting the Operational Evaluation}

The challenges involved in achieving readiness for an airline operational evaluation of TASAR were detailed in previous publications. [5] [13] This section will discuss the challenges of conducting the operational evaluation and the approach selected to address these challenges.

\section{A. TAP Operators}

The challenges of conducting an effective operational evaluation centered primarily on the ability to collect a sufficient quantity of data (both in terms of quantity and quality) using only the three TAP-equipped aircraft. To collect sufficient data during the evaluation period it was necessary to have a TAP-trained pilot onboard each of the three TAP-equipped aircraft as often as possible. Because the logistics would be extremely difficult to manipulate the schedule of the three TAP-equipped aircraft as well as the crews assigned to flights on those specific aircraft, only a small fraction of Alaska pilots would have the opportunity to use the TAP software application during the evaluation period. Considering that Alaska operates a fleet of over 150 Boeing 737 aircraft and has over 1500 active pilots, it could be many months between TAP flights for any given TAP-trained pilot. Additionally, knowledge and proficiency deteriorate over long periods of non-use, and so rolling out a training program to the entire pilot group was not a 
reasonable approach. In light of these challenges, as well as the substantial cost of training pilots, it was deemed impractical to train all Alaska pilots on the TAP application for a limited operational evaluation.

Important to the success of this operational evaluation was to collect data on as many flights as possible and across a diverse set of routes. Initial assessments of TASAR benefits, using historical flight data in a fast-time simulation environment, indicated that the benefits would likely vary depending on stage length (i.e., origin to destination distance) and destination airport. [6] [8] In general, longer flights into non-hub airports (i.e., those with fewer ATC flow restrictions) are better suited to enabling TASAR benefits. Whereas the airline could have tried to dedicate the three TASAR aircrafts to these types of routes, it was logistically unrealistic to do so without disrupting the network efficiency of the airline. Moreover, matching the schedules of specific TAP-trained pilots (assuming the entire pilot population could not be trained) to the schedules of these particular aircraft, regardless of route, would have nearly impossible logistics.

An approach was therefore devised to use trained Alaska employees as "TAP Operators" and have them operate TAP from the cockpit jump seat. The approach addresses the challenges described above and achieves the objective of maximizing the number of TASAR flights achieved within reasonable cost and logistical constraints. The following sections discuss TAP Operator training, in-flight procedures, and subjective data collection methodology.

\section{TAP Operator Training}

Specific individuals were identified by Alaska to serve as TAP Operators to include three Alaska interns and four Alaska Technical Pilots. The TAP Operators were provided with training materials: the TAP Operating Procedures Handbook, the TAP Computer Based Training Module, and the supplemental TAP Flight Manual Bulletin. Additionally, NASA TASAR researchers provided the TAP Operators with two days of in-person classroom training on the TASAR concept and the procedures, features, and operation of the TAP software application. Training included hands-on use-case examples and use-case dry runs in the Alaska flight simulator. Finally, the interns flew with Alaska Technical Pilots on one or two training flights to become familiar with the cockpit jump-seat procedures as well as the operation of TAP during actual flights.

\section{TAP Operator In-Flight Procedures}

To ensure consistency during data collection and to provide the TAP Operators with specific procedures to follow during the TASAR flights, a set of instructions and procedures were developed in the form of checklists (Figure 3). The checklists covered all procedures before, during, and after the flights to help with proper configuration and usage of TAP. While the TAP Operator was the trained user of TAP, they engaged the flight crew on TAP's route modification advisories and encouraged their consideration by the crew for an ATC request. Flight crews were given the opportunity to see and interact with TAP during the flight. When a TAP route modification was selected for a request, the flight crew used normal cockpit procedures and phraseology in making the request to ATC. If the request was approved, the flight crew changed the aircraft's route and/or altitude according to the new ATC clearance, and the TAP Operator recorded the ATC approval in TAP. As the flight continued, TAP would monitor for more route optimization opportunities, and any further opportunities were handled with the same procedure. TAP use was discontinued prior to the flight crew preparing for arrival at the destination airport.

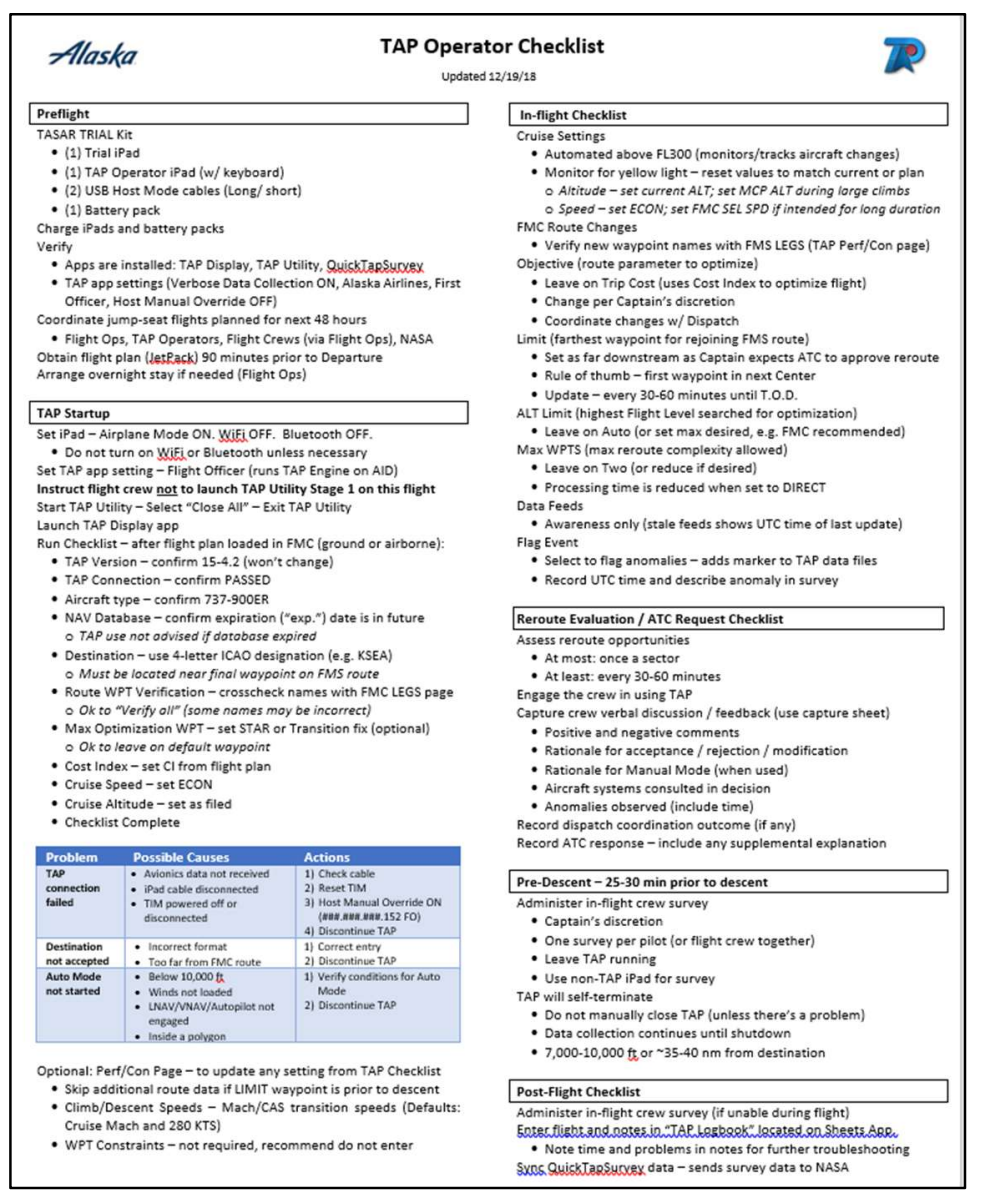

Figure 3. TAP Operator checklist. 


\section{TAP Operator Subjective Data Collection}

The TAP Operators were asked to complete surveys during and after each TASAR flight so that the research team could fully understand the events that occurred during the flight when conducting data analysis. These surveys included the TAP Operator Capture Sheet and the TAP Operator Weekly Report. Additionally, the flight crew was asked to complete a TAP Crew Survey if the crew interacted with TAP during the flight. These surveys were administered via the QuickTapSurvey app on the TAP Operators iPad.

The main purpose of the TAP Operator Capture Sheet was to collect data regarding TAP-generated advisories and the events that occurred when the TAP Operator presented the TAP advisory to the crew. This enabled post-flight analysis to determine what percentage of the TAP-generated route modifications were reviewed and requested by the flight crew to ATC, the ATC response, and subsequent flight crew action.

The TAP Operator Weekly Report was designed to capture the subjective opinions of the TAP Operators after they completed several flights during a week. Questions included topics such as perceived acceptability of the TAPgenerated advisories, quality and accuracy of the TAP computed fuel/time outcomes for advisories, TAP problematic behavior/potential software bugs, TAP system usability, flight crew interactions, and materials/procedures recommendations. The intent of this survey was to gather information based on the TAP Operators' cumulative experience to improve the data collection process as well as address any issues with TAP. Presentation of surveybased subjective data analysis from the operational evaluation is planned for future publications.

The approach of using TAP Operators flying in the jump seat during initial TASAR flights was critical to the success of the operational evaluation. This approach allowed for the collection of substantially more data than otherwise would have been logistically possible given that, unlike the flight crew, the TAP Operators were unconstrained by duty day limitations or scheduling challenges. Additionally, the approach allowed technical issues to be efficiently addressed without disturbing the flight crew. Because the TAP Operators on these initial flights were not pilots actively flying the aircraft, they could focus on documenting the issue, as well as spend time troubleshooting that would not have been appropriate for the pilot flying. As a result, several very successful fixes and modifications were made that improved the use of TAP for later flights. Since the underlying trajectory generator and route optimizer algorithm were not modified throughout the evaluation period, data from before and after the software updates were included in a single analysis.

\section{B. TAP Flight Route Selection and Balancing}

The Boeing 737-900ER was selected for this operational evaluation in part because it typically flies the longerdistance routes which analysis has shown are better suited for the TASAR operation. Though airlines will be interested in understanding which city pairs may benefit the most from TASAR, it was not possible to schedule the three TAPequipped aircraft to fly between specific city pairs frequently enough during the evaluation period to produce a large enough sample size to achieve statistical significance. A modified zone approach was used wherein airports with similar characteristics were collected into zones as shown in Figure 4.

Ten zones were defined to consolidate airports that, when flown as "zone pairs," were expected to have similar characteristics from the perspective of TASAR benefits. For instance, since aircraft flying from Zone 0 to Zone 9 are all transcontinental flights, they would have exposure to similar national weather patterns, and they would not be subjected to airspace restrictions associated with the northeast region (Zones 7 or 8).

Logistically, zone-pair association also provided flexibility for aircraft swaps. It is not uncommon for a specific aircraft assigned to a particular flight to be swapped for another aircraft for a variety of reasons (e.g., maintenance delays, load factor optimization, delay management logistics). Such swaps could have been detrimental for the TASAR operational evaluation if it

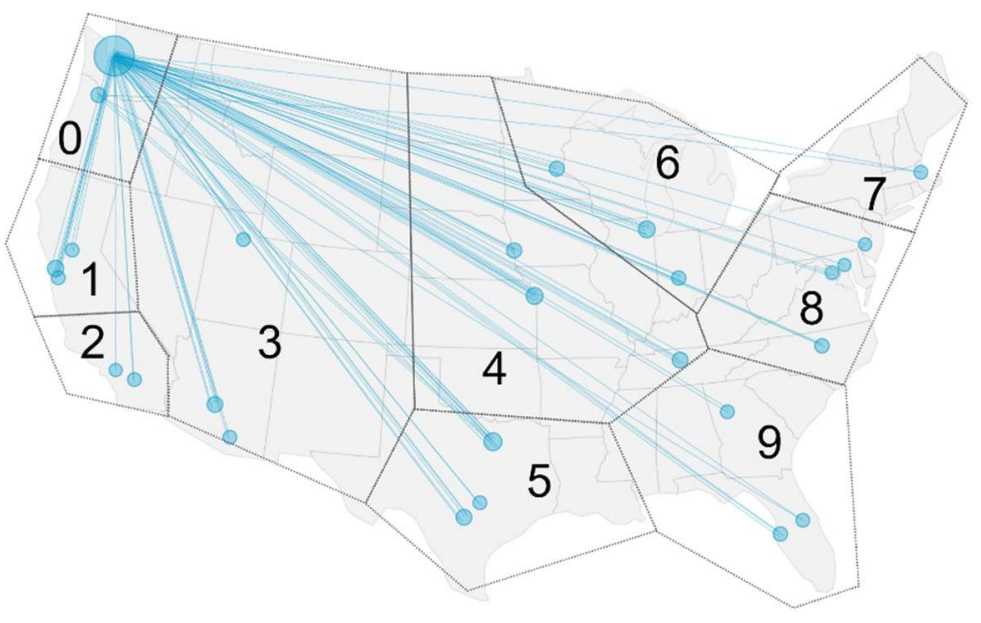

Figure 4. Assignment of Continental US airports to ten zones. Each line represents a valid TAP flight between airports (depicted as circles) flown during the Alaska operational evaluation. 
disrupted plans for a TAP Operator to be positioned to board a TAP-equipped aircraft for a predetermined itinerary. However, to the extent the equipment swap could keep the TAP-equipped aircraft flying the same zone pair, the new itinerary will bear enough resemblance to the original such that the TAP Operator logistics, as well as the likely benefit opportunities, should be similar enough to not impact data collection.

Due to a shortened evaluation period, an insufficient number of flights were achieved to permit statistical analysis of benefits by zone pair. Generally, the largest benefits occurred on mid-continental and transcontinental flights, as was expected.

\section{Benefits Assessment Methodology and Initial Results}

In order to quantitatively estimate achieved TAP benefits, data were recorded by TAP during operational flights and analyzed post-flight. TAP-recorded data included aircraft systems data and pilot interactions with TAP. TAP benefits were calculated as the cost difference between TAP-flight operating costs and baseline-flight operating costs. During TAP flights, TAP-computed route-modification advisories were displayed to pilots and used to make "TAPinspired" route-modification requests to ATC. Data on pilot interactions with TAP were used to determine whether or not a route modification observed in the aircraft systems data was due to TAP (i.e., TAP-inspired). Baseline flights also had TAP running onboard but was not used by pilots or TAP Operators (i.e., TAP was running automatically in a mode with no user interface). During these baseline flights, TAP recorded "as flown" aircraft data and computed route-modification advisories, but the advisories were not requested to ATC or flown. The TAP data collection on these "non-TAP flights" served as baseline flights in the benefits analysis.

Fuel and time savings were converted to cost savings in U.S. dollars (\$). Typically, airlines use an hourly directoperating-cost parameter, which includes fuel cost, to convert time savings to cost savings. However, since TAPinspired altitude changes traded off between fuel and time, the analysis separated the time-related and fuel-related components of hourly direct operating costs. Alaska's hourly direct operating costs $(\$ 1,710 /$ hour excluding fuel) and fuel costs (\$2.28/gallon) used in this analysis were estimated from third quarter 2018 financial reports (Form 41, Schedule P5.2) obtained from the Bureau of Transportation Statistics. [14]

In Eq. (1), which represents achieved TAP benefits, the first term to the right of the equals sign is the difference between flown aircraft cost $\left(C^{\text {flown }}\right)$ and unimpeded predicted aircraft cost $\left(C^{\text {predicted }}\right)$ on TAP flights. The second term similarly shows this cost difference corresponding to non-TAP baseline flights. Flown costs are incorporated into the equation since unpredictable events may occur after TAP-inspired requests. These events may include pilot and ATC actions required to meet their objectives as well as changing atmospheric conditions. Flown fuel and time are measured relative to fuel and time predictions generated just prior to the TAP request to reduce the impact of route and atmospheric differences between flights with TAP operators and the baseline flights.

$$
\text { Total Cost Change }=\sum_{T A P \text { fligh }}\left(C^{\text {flown }}-C^{\text {predicted }}\right)-\sum_{\text {baseline flights }}\left(C^{\text {flown }}-C^{\text {predicted }}\right)
$$

Section III.B reports results using both the flight plan and TAP for $C^{\text {predicted }}$ since there are advantages and disadvantages to using either prediction. Flight plans predict step climbs and are used operationally at Alaska. However, aircraft may be off their pre-departure flight plan at the time a TAP advisory is approved. TAP does not predict step climbs but TAP predictions reflect updated route and wind information received post-departure.

\section{A. Method}

\section{TAP flight cost}

To quantify the impact of TAP on a flight, the flown segment altered by TAP-inspired route modification requests was identified as follows. The start of the segment is referred to as the TAP Start Point (TSP) which is the location of execution of the first executed TAP advisory as shown in Figure 5. The end of the segment is referred to as the TAP Finish Point (TFP) which is the location where the aircraft rejoins the flight plan route after completing the final executed TAP advisory. Multiple TAP-inspired route modifications may occur between the TSP and TFP. The TFP defaults to Top-of-Descent (TOD) if the altitude or route of the as-flown TAP flight did not rejoin the pre-departure flight plan altitude or route prior to TOD. For analysis purposes, it is assumed that the portions of the flight before the TSP and after the TFP were not affected by TAP.

After identifying TSP and TFP, the cost difference $\left(C^{\text {flown }}-C^{\text {predicted }}\right)$ is obtained by subtracting the predicted fuel and time at TFP from the closest ownship state fuel and time at TFP and converting to cost. Since both $C^{\text {flown }}$ and $C^{\text {predicted }}$ begin at a common point (TSP), the cost at TSP cancels out in the equation and only the flown and predicted cost at TFP is used when calculating the cost difference. If necessary, the pre-departure flight plan and/or TAP predicted fuel and time at TFP were obtained using interpolation. 


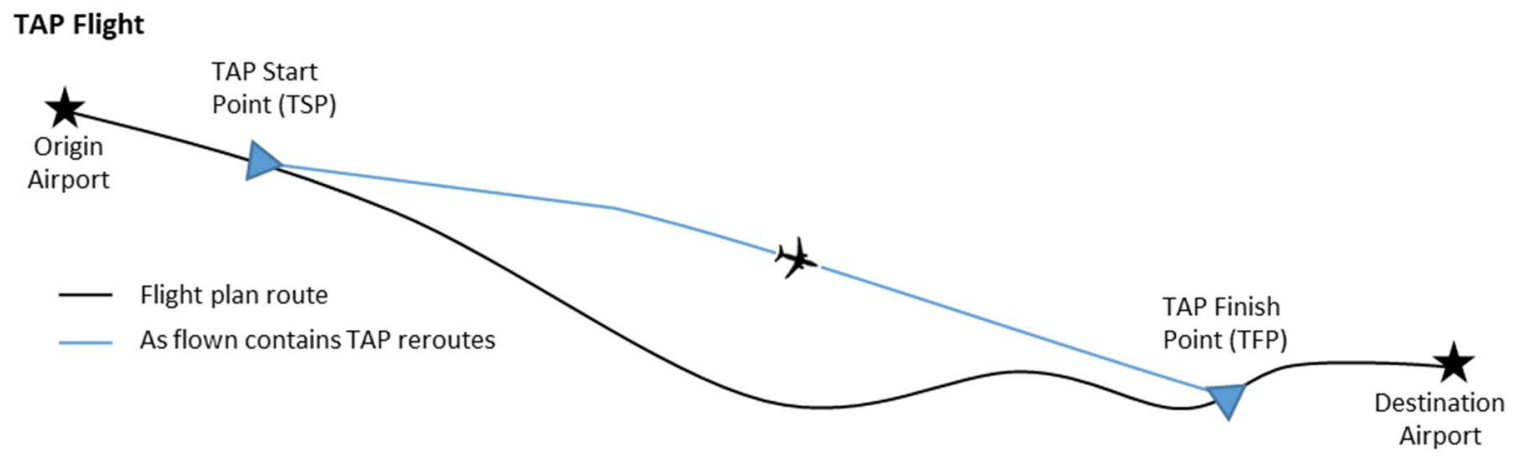

Figure 5. TAP Start Point (TSP) to TAP Finish Point (TFP) segment impacted by TAP-inspired requests. Note that the aircraft may be off its pre-departure flight plan at the TSP and/or TFP.

Note that whether TAP-inspired requests were executed cannot be known with certainty since pilots manually enter ATC-approved TAP-inspired requests into the FMS. For this operational evaluation, TAP did not have the capability to auto-load ATC-approved advisories to the FMS, and therefore no data records reliably link a TAP advisory to an actual route modification. One example of TAP-inspired requests that are difficult to detect are partially executed combination (lateral and altitude) changes. The controller may immediately approve the altitude change but withhold approval of the lateral path change. Several minutes later the controller may then approve a lateral path change that is similar to the lateral path component of the TAP advisory. Since the lateral path change did not occur at the same time as the altitude change, it was challenging in some cases to determine whether the lateral path change was due to a TAP-inspired request. Two complementary data sources were used to reduce the chance of mischaracterizing executed TAP-inspired requests: (1) TAP data recording of pilot interactions with the TAP Display, including pilot-selected TAP advisories and pilot-recorded (via the TAP Display) ATC approval, and (2) a post-flight TAP logbook containing TAP operator observations. However, both data sources may not have contained complete information since performing these actions was not required.

\section{Baseline cost}

After the TSP and TFP were identified on the TAP flight, they served as benchmark locations for the corresponding non-TAP baseline flight. A corresponding Baseline Start Point (BSP) and Baseline Finish Point (BFP) along the predicted path of the baseline flight shown in Figure 6 was selected as follows. To simplify the analysis, the BFP was selected to be at the flown TOD since the TFP was generally at or near the TOD. The TFP was at the TOD 66\% of the time when using flights plans to compute $C^{\text {predicted }}$. The TFP was at the TOD $100 \%$ of the time when using TAP data to compute $C^{\text {predicted }}$. The BSP was selected so that $C^{\text {predicted }}$ from BSP to BFP was as close as possible to $C^{\text {predicted }}$ from TSP to TFP. Sampling both TAP flights and the non-TAP baseline flights matched to these flights are described next.

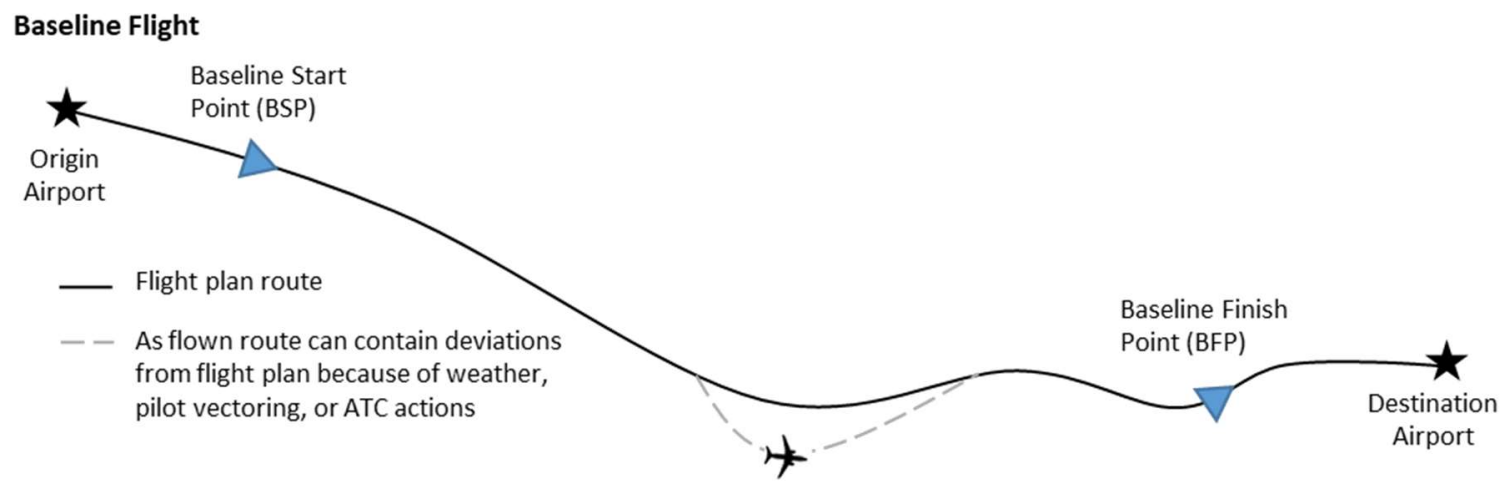

Figure 6. Baseline Start Point (BSP) to Baseline Finish Point (BFP) baseline segment. Note that the aircraft may be off its pre-departure flight plan at the BSP and/or BFP. 


\section{Flight sampling}

On certain flights, the use of TAP was limited due to certain technical integration issues and limitations associated with the operational evaluation (e.g., navigation database limited to U.S. airspace). For this reason the validation criteria in the left column of Table 1 was used to exclude, for example, TAP flights where pilots did not use TAP within the Continental US (CONUS) for a sufficient period of time. Benefits were calculated for all flights meeting the validation criteria regardless of other flight characteristics.

For each valid TAP flight a baseline flight was selected as follows. Valid TAP flights with an approved TAPinspired route modification request were ordered by date. Valid TAP flights without an approved TAP-inspired route modification request were included in the results as zero benefit but did not require a corresponding baseline flight. Starting with the first valid TAP flight, the set of baseline flights meeting the criteria in the right column of Table 1 were used as candidates to be paired with a TAP flight. The candidate baseline flight that occurred closest in calendar date to the TAP flight (to mitigate seasonal variations) was matched to the TAP flight and removed as a pairing candidate for other TAP flights. This proceeded sequentially until all valid TAP flights with an approved TAP-inspired route modification request had a corresponding baseline flight.

The operational evaluation was conducted between July 24, 2018 and April 30, 2019. The following values and the benefit results presented in this paper are preliminary, based on the subset of data available at publication. Based on this subset, a total of 119 evaluation flights were reviewed for analysis, including 70 flights between July 24 and September 20, 2018 and an additional 49 flights between January 23 and April 30, 2019. Eighty-three of those flights were determined to be valid according to Table 1 criteria. Of those 84 valid flights, 54 (64\%) were determined to have had an approved TAP-inspired request during the flight.

Table 1. Criteria for TAP flights to be included in benefit set (left column) for a baseline flight to be considered a candidate for matching to a TAP flight (right column). Note that the "advisory generation state" occurs when the pilot completes the checklist page and TAP receives sufficient data to calculate advisories.

\begin{tabular}{|c|c|}
\hline TAP Flight Validation Criteria & Baseline Flight Candidate Criteria \\
\hline Departure airport is within CONUS & Departure airport is within CONUS \\
\hline Arrival airport is within CONUS & Arrival airport is within CONUS \\
\hline $\begin{array}{l}\text { Flight time above FL1 } 80 \text { within CONUS must be at least } \\
75 \% \text { of total flight time from wheels-off to wheels-on }\end{array}$ & \multirow{2}{*}{$\begin{array}{l}\text { Flown flight duration is within the same range as } \\
\text { TAP flight: } \\
\text { 1) } 0 \text { to } 2 \text { hours } \\
\text { 2) } 2 \text { to } 4 \text { hours } \\
\text { 3) More than } 4 \text { hours } \\
\text { e.g., if TAP flight duration is } 3 \text { hours then baseline } \\
\text { flight must have been } 2 \text { to } 4 \text { hours long. }\end{array}$} \\
\hline $\begin{array}{l}\text { At least one pilot interaction with the TAP Display (i.e., } \\
\text { button press) occurred while TAP was in an advisory } \\
\text { generation state }\end{array}$ & \\
\hline $\begin{array}{l}\text { At least one of the following criteria is met: } \\
\text { 1) Pilots made a TAP-inspired request to ATC } \\
\text { 2) Cumulative } 30 \text { minutes in advisory generation } \\
\text { state } \\
\text { 3) } 50 \% \text { of the flight time above FL180 in advisory } \\
\text { generation state }\end{array}$ & $\begin{array}{l}\text { TAP on the baseline flight was making ownship } \\
\text { predictions and recording ownship state for at least } \\
\text { the predicted time duration from TSP to TFP on the } \\
\text { TAP flight (i.e., exclude baseline flights that were } \\
\text { not recording data for a sufficient period of time). }\end{array}$ \\
\hline TAP-recorded data was retrieved from the aircraft & TAP-recorded data was retrieved from the aircraft \\
\hline
\end{tabular}

\section{B. Initial Results}

Table 2 summarizes benefits for all TAP flights regardless of flight length. The first row shows the summation of the flown cost minus the predicted cost across all valid TAP flights. On average, flown cost was higher than predicted cost since both the flight plan predictions (middle column) and TAP predictions (rightmost column) are unimpeded predictions, and unplanned deviations were common on most flights (e.g. ATC vectors, maneuvering for weather). The second row similarly shows this summation for baseline flights. The total cost change is shown in the third row, which is calculated as the first row minus the second row. Since there was a reduction in cost attributed to TAP, the cost change is multiplied by -1 to provide the total benefit in row 4 . Row 4 is divided by the number of valid flights (84) to obtain the average cost savings per flight based on flight plan predictions ( $\$ 92.08 /$ flight) and TAP predictions (\$87.60/flight). The standard deviation (std dev) and margin of error (MoE) are also shown to indicate the spread of 
flight-by-flight benefits and the confidence interval around the benefit at $80 \%$ and $95 \%$ confidence levels. The time and fuel benefit estimations are then shown in the final two rows.

Baseline flights were successfully collected during the July 24 to September 20, 2018 time period. However, technical issues prevented the baseline flight data collection during January 23 to April 30, 2019. In a conservative approach, the TAP flights during this time period instead were paired with baseline flights from winter 2018-19 (September 2018 to January 2019) to estimate the benefits shown in Table 2. To characterize the sensitivity of benefit estimation to the selection of the baseline, benefits based on baseline flight data from spring 2018 (January to July) and summer 2018 (July to October) were calculated using both the flight plan and TAP prediction methods. Average cost savings per flight for the flight plan predictions ( $\$ 104.39$ /flight for summer vs. $\$ 92.08 /$ flight for winter) and the TAP predictions (\$107.16/flight for spring to $\$ 108.18$ /flight for summer vs. $\$ 87.60$ /flight for winter) were similar but less conservative when using spring and summer 2018 baselines as compared to the winter 2018-19 baseline.

Data had not been received from six flights in time to be included in the final version of this paper. For this reason, and to refine the analysis of baseline flights, benefit results reported in Table 2 will be updated in a final report. [14]

A fast-time simulation analysis previously estimated the benefits of using TAP on Alaska aircraft. [8] This study, which was based on data from 2012 when there were few high impact convective weather events, found that in excess of $90 \%$ of predicted benefits can be attributed to a lateral change to a more wind optimal trajectory. This is in contrast to the operational evaluation, where $69 \%$ of flights with approved requests ( 37 of 54 flights) and $83 \%$ of total approved requests (101 of 122 requests) were for altitude changes only.

Table 2. Aggregate benefit results corresponding to 84 analyzed valid flights.

\begin{tabular}{|c|c|c|}
\hline Item & Flight Plan $C^{\text {predicted }}$ & TAP $C^{\text {predicted }}$ \\
\hline$\sum_{\text {TAP flights }}\left(C^{\text {flown }}-C^{\text {predicted }}\right)$ & $\$ 703.65$ & $\$ 2,352.01$ \\
\hline$\sum_{\text {baseline flights }}\left(C^{\text {flown }}-C^{\text {predicted }}\right)$ & $\$ 8.437 .96$ & $\$ 9,710.68$ \\
\hline Total cost change due to TAP $(\$)$ & $-\$ 7,734.31$ & $-\$ 7,358.67$ \\
\hline Total benefit (\$) & $\$ 7,734.31$ & $\$ 7,358.67$ \\
\hline Benefit per valid flight (\$/flight) & $\begin{array}{l}\$ 92.08 / \text { flight } \\
\text { std dev }=\$ 270.46 \\
\mathrm{MoE}=\$ 37.82, \text { CL } 80 \% \\
\mathrm{MoE}=\$ 57.84, \text { CL } 95 \%\end{array}$ & $\begin{array}{l}\$ 87.60 / \text { flight } \\
\text { std dev }=\$ 264.04 \\
\mathrm{MoE}=\$ 36.92, \text { CL } 80 \% \\
\mathrm{MoE}=\$ 56.46, \text { CL } 95 \%\end{array}$ \\
\hline $\begin{array}{l}\text { Benefit per valid flight } \\
\text { (minutes/flight) }\end{array}$ & $\begin{array}{l}0.82 \text { minutes/flight } \\
\text { std dev }=3.87 \text { minutes } \\
\mathrm{MoE}=0.54 \text { minutes, CL } 80 \% \\
\mathrm{MoE}=0.83 \text { minutes, CL } 95 \%\end{array}$ & $\begin{array}{l}0.46 \text { minutes/flight } \\
\text { std dev }=4.45 \text { minutes } \\
\mathrm{MoE}=0.62 \text { minutes, CL } 80 \% \\
\mathrm{MoE}=0.95 \text { minutes, CL } 95 \%\end{array}$ \\
\hline Benefit per valid flight (gallons/flight) & $\begin{array}{l}\text { 30.2 gallons/flight } \\
\text { std dev }=88.4 \text { gallons } \\
\mathrm{MoE}=12.36 \text { gallons, CL } 80 \% \\
\mathrm{MoE}=18.90 \text { gallons, CL } 95 \%\end{array}$ & $\begin{array}{l}32.7 \text { gallons/flight } \\
\text { std dev }=81.4 \text { gallons } \\
\mathrm{MoE}=11.38 \text { gallons, CL } 80 \% \\
\mathrm{MoE}=17.41 \text { gallons, CL } 95 \%\end{array}$ \\
\hline
\end{tabular}

The simulated Alaska 737-900 aircraft in the fast-time simulation analysis had the closest routes to those flown during the operational evaluation. The predicted TAP benefit corresponding to these aircraft was about 25 gallons/flight and 2.8 minutes/flight corresponding to a cost savings of $\$ 136.05 /$ flight. TASAR flights during the operational evaluation period achieved estimated benefits of 30.2 gallons/flight and 0.82 minutes/flight of savings, representing higher fuel savings, lower time savings, and lower cost savings at $\$ 92.08 /$ flight than predicted by the fast-time simulation. However, there is uncertainty in these estimates due to the small sample size of operational evaluation flights included in the analysis.

It is possible that additional benefits would have been achieved if TAP had generated, and TAP Operators had selected, more advisories to follow a more wind optimal trajectory, but this behavior was not frequently observed. The reason could be shifts in post-departure wind direction and magnitude relative to the pre-departure flight plan route during the operational evaluation being less conducive to lateral wind optimization than was predicted by fasttime simulation. It is also possible that the lower fidelity fast-time simulation identified wind cases that the higher fidelity TAP automation correctly identified as being non-beneficial. An additional factor may have been the interactions between the flight crews and the TAP Operators in the jump seat in the operational evaluation. The TAP 
Operators may have been more successful in proposing altitude changes to the flight crew than they were in proposing lateral route modifications, given that altitude changes are more easily requested to ATC in a voice environment.

The items in Table 3 were identified as factors indicating the average benefits estimated per flight in the operational evaluation (\$92.08/flight) may be a conservative estimate:

Table 3. Factors that may indicate benefits measured in operational evaluation are conservative.

\begin{tabular}{|c|l|}
\hline 1 & TAP was rarely used as intended (i.e., by the flight crew flying the aircraft) \\
\hline 2 & Interns were non-airline pilots and, being junior to the flight crew, had limited influence on decisions \\
\hline 3 & All TAP Operators had limited experience with TAP and its use in operations (learning curve effect) \\
\hline 4 & Flight crews (working with trained TAP Operator riding in the jump seat) had no TAP training \\
\hline 5 & Not all actual TAP benefits were measurable in this evaluation (e.g. plan validation benefits) \\
\hline 6 & Not all benefit opportunities identified by TAP were executed (i.e., either not requested or not approved) \\
\hline 7 & Fewer lateral and combo TAP advisories were executed compared to the pre-trial estimate \\
\hline 8 & Short flights (<2 hours) had few benefit opportunities, but were easier to schedule and therefore included \\
\hline 9 & Benefit results included flights w/ zero benefit, which reduced the per-flight average across all flights \\
\hline 10 & Winter baseline flights (for springtime TAP flights) were less affected by convection (i.e., more efficient) \\
\hline 11 & TAP was an initial prototype (i.e., technical improvements are expected in commercial TAP versions) \\
\hline
\end{tabular}

To indicate the potential magnitude of the effect of the first two items in this list, the average benefits were by type of TAP Operator were calculated. The benefit per flight where the TAP Operator was an intern was \$42.70/flight. In comparison, the benefit per flight where the TAP Operator was either an Alaska Airlines technical pilot or a NASA researcher (i.e., highly experienced with TAP) was $\$ 173.64 /$ flight, or over four times larger than the benefits achieved on intern TAP Operator flights.

A contrary factor indicating the benefits may be a non-conservative estimate was that every TAP flight in the operational evaluation included an enthusiastic TAP user in the cockpit. Not all flight crews necessarily embrace new technology, and even for those who do, the regular use of TAP in all opportunities would require a culture change that will take time.

\section{Categories of Operational Benefit}

Figure 7 depicts the distribution of achieved benefits for the 84 valid TAP flights of the Alaska operational evaluation. The horizontal axis is the benefit in dollars, with each bar having a width of $\$ 50$. The vertical axis represents the number of flights achieving the benefit. A kernel density estimate (KDE) curve fit is presented in blue.

The distribution of achieved benefits suggests three distinct benefit categories: Home Runs (A), Cumulative Small Gains (B), and Plan Validation (C). The cost savings provided by Home Runs (A) are significant but occur infrequently. Using $\$ 400$ savings as a demarcation, seven of 84 flights $(\sim 8 \%)$ achieved benefits in this category. Cumulative Small Gains (B) are evident in the non-symmetric "bump" in the curve fit on the positive side of the vertical axis, where for example eight flights each achieved approximately $\$ 300$ benefit. Plan Validation (C) is indicated by 34 flights with negligible or zero benefits (including 30 flights making no TAP-inspired requests). This "zero" bar represents a combination of flights with no opportunity for improvement and those where the flight crew may have used TAP to avoid making an unintentionally detrimental request. The region to the left of the vertical axis reflect unrelated route changes that offset TAP gains. Most flights experience disruptions for various reasons such as conflict resolution instructions by ATC, maneuvering by pilots for weather avoidance, and altitude changes requested by pilots for turbulence mitigation. In some situations, a fourth benefit category, Improved Situation Awareness (D) through TAP, could help minimize the cost of such disruptions. The sections below give example cases of these benefit categories observed in the Alaska operational evaluation.

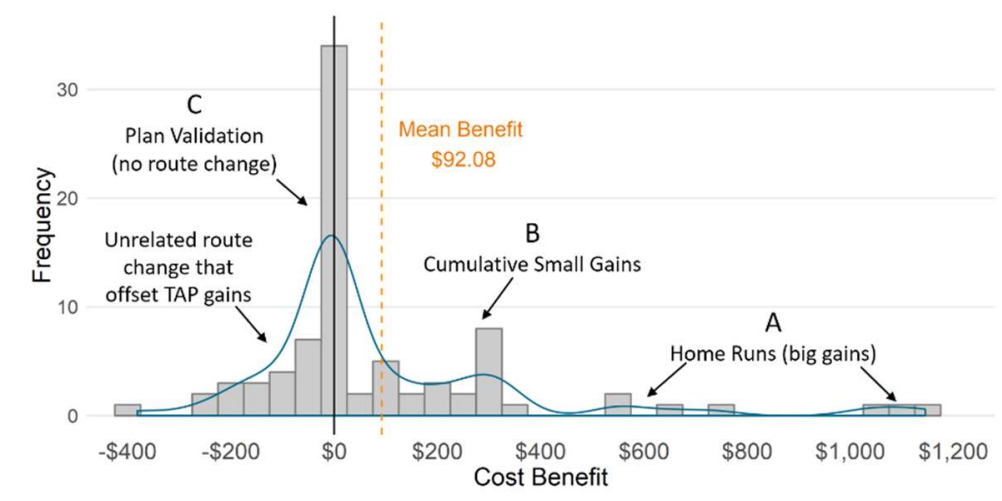

Figure 7. Distribution of achieved benefits of TAP flights. 
It is useful to note that these trends emerged even under the limited number of flights of the operational evaluation and with the limited experience TAP Operators had with this new technology. The full potential for achieving TASAR benefits is driven by three factors: opportunity, action, and approvability. The opportunities for route improvement must exist, action must be taken by the flight crew to request a route modification, and the request must be approvable (and actually approved) by ATC to be executed. As an optimization tool, TAP is designed to address the first and third factors directly: it monitors the flight vigilantly for beneficial route modification opportunities, and it increases approvability by accounting for ATC constraints such as nearby traffic in its advisories. TAP indirectly addresses the second factor - action - by incorporating human-centered principles in the user interface design to maximize system usability. But action will also be driven over time by accumulated experience using TAP and a culture change wherein flight crews engage more frequently and proactively in route optimization throughout the flight and further integrate technology such as TAP into that role. TASAR benefits are expected to increase as this culture change unfolds. The following sections present example cases of benefit categories observed in the Alaska operational evaluation.

\section{A. Home Runs}

The benefit category that provides the greatest benefit in operational cost savings (time and fuel) is the "Home Runs" category. This category reflects those opportunities where a significant improvement to the aircraft's route is possible relative to the flight plan route or a modified weather routing issued by ATC. Flight planning systems generally do an excellent job of creating efficient routes, taking into account forecast weather available at the time. However, forecast inaccuracies and conservative planning can result in occasions where the expected conditions differ significantly from the actual conditions experienced in flight. Similarly, ATC may issue conservative "playbook" routing around weather when significant en route weather is forecast, but actual conditions may be less severe and permit opportunities for improved routing. TASAR offers significant benefits in such scenarios through monitoring the latest weather and wind information and continually refreshing the search for more efficient route opportunities.

An example of the Home Runs benefit category occurred on an Alaska TAP flight in July 2018 from San Antonio to Seattle. The flight plan routed the aircraft conservatively north of Denver to account for weather on the direct course. On the climb out of San Antonio, TAP received updated winds and weather data and computed a "combo" (lateral plus vertical) route modification advisory. As shown in Figure 8, the weather development (indicated by blue polygons) provided a number of shorter routing opportunities through large gaps in the weather (note the display scale of 1340 nmi), including the lateral path identified by TAP (direct from present position to the Donnelly waypoint, DNJ). Vertically, TAP also made the recommendation to climb to Flight Level (FL) 340, rather than leveling at FL320 per the flight plan. Together, these lateral and vertical changes, if flown without change, were predicted by TAP to save over $1800 \mathrm{lbs}$ fuel and nearly 13 minutes flight time. The pilot made the request to ATC, approval was received, and the route modification was executed.

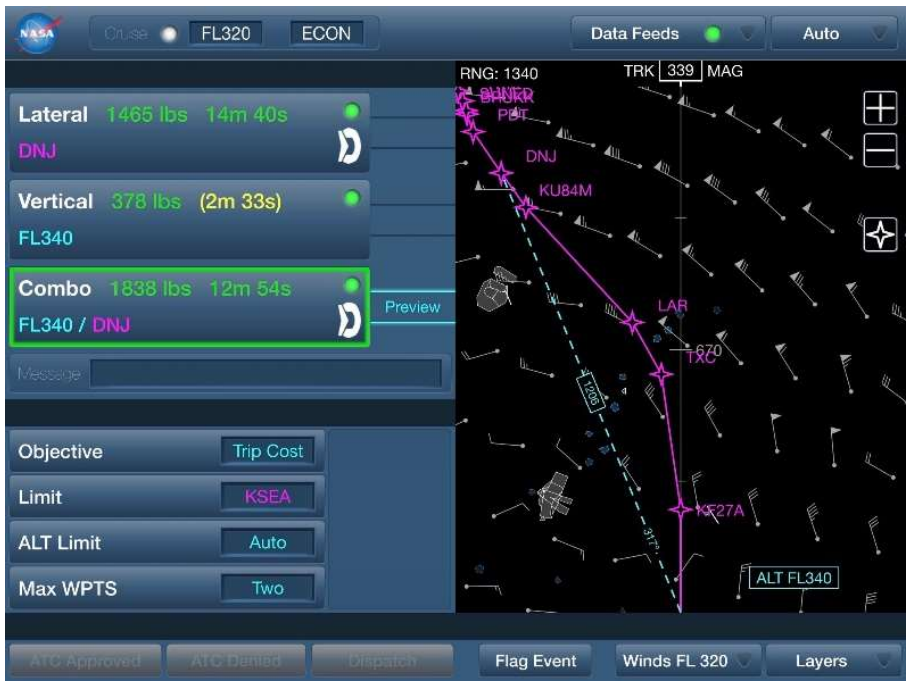

Figure 8. Example of "Home Runs" benefit category showing a combination lateral/vertical route modification with significant fuel and time savings.

\section{B. Cumulative Small Gains}

The second TASAR benefit category generates savings on a much smaller scale per individual instance but may aggregate into significant cumulative savings for the airline through multiple requests on a given flight and across many flights. These "Cumulative Small Gains" leverage the opportunity of a flight crew to "fine tune" the route during periods of low workload (often the majority of the en route segment). The flight crew minimizes impacts to ATC workload by monitoring the frequency to avoid congested periods and making at most one request per sector. TAP remains vigilant for benefit opportunities no matter how large or small. Since TAP has no minimum threshold for savings when presenting route modification options to pilots, the flight crew will have the opportunity to act upon even minor route modification advisories at their discretion. Such opportunities can emerge, for example, when TAP receives a wind update (an hourly occurrence during this operational evaluation). The updated winds, which can be 
several hours more recent than winds used by the flight planning system, will occasionally favor an earlier or delayed climb to a higher altitude or a nondirect route between flight plan waypoints.

An example of the latter case occurred on an Alaska TAP flight in July 2018, also from San Antonio to Seattle. While en route, the aircraft was on a northwesterly direct route to the Pendleton waypoint, PDT, facing headwinds initially from the northwest switching to westerly downstream. Set to optimize for fuel burn and taking recently published winds into account, TAP computed a non-direct lateral solution through the EDLES intersection that would save $102 \mathrm{lbs}$ fuel and 61 seconds flight time (see Figure 9). The pilot requested the route modification and it was approved. Though the benefit was modest compared to the "Home Run" example, multiple such requests made by pilots on all TAP-equipped flights would accrue over time

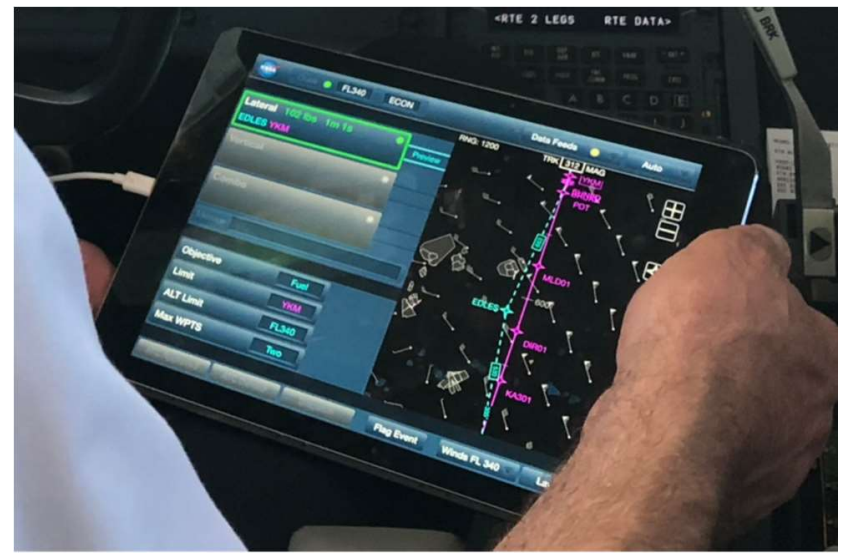

Figure 9. Example of "Cumulative Small Gains" benefit category showing a modest wind-optimized lateral route modification to the left of the current course. into a more significant fleet-wide savings.

\section{Plan Validation}

The existence of the third benefit category, "Plan Validation," became apparent during the operational evaluation as pilots used TAP to consider and then reject a prospective change from the flight plan. The benefit, in this case, is not the generation of savings through identifying and executing an improvement to the current route. Rather, the benefit is in preempting an inadvertent negative benefit (i.e., cost impact) that would have resulted from an uninformed route modification decision by the flight crew. It is common for flight crews to make occasional requests to ATC to achieve a seemingly more optimal route using rules of thumb and optimization information available from onboard information. The "up and straight" philosophy can lead pilots to seek routing short cuts and climbs to the optimum altitude indicated by the FMS at the earliest opportunity, despite the recommendations of the flight plan. However, such decisions can sometimes be detrimental to optimization.

An example of Plan Validation occurred on a February 2019 TAP flight from Seattle to Palm Springs International. The aircraft was level at the flight plan's initial cruise altitude of FL350. No TAP advisories were actionable (lateral, vertical, or combo), despite the FMS indicating FL370 as the optimal altitude. The flight plan indicated that the aircraft should remain at a cruising altitude of FL350 for the flight. To assess the impact, the pilot entered FL370 into TAP's manual mode as a candidate route modification. TAP confirmed the maneuver would be detrimental to fuel burn and flight time, and therefore the pilot remained at the filed altitude of FL350, having used TAP to validate the flight plan. TAP's knowledge of the three-dimensional wind model enabled it to integrate the effect of the less favorable winds at the higher altitude through the full trajectory and effectively preempt a decision by the pilot to climb to the FMS optimum altitude.

\section{Improved Situation Awareness}

An additional utility of TASAR emerged serendipitously on multiple evaluation flights - improved situation awareness. Though not a primary benefit mechanism wherein TAP scans for route optimization opportunities and displays these to the flight crew, it assisted pilots in proactive decision-making, sometimes resulting in quantifiable operational efficiency benefits. TAP's manual mode was frequently germane to this use case, as was shown in the previous example for "Plan Validation." Manual mode facilitates the evaluation of "what if" scenarios by enabling the pilot to enter a proposed route modification and having TAP predict the effects on fuel burn and flight time, while also identifying any interactions with nearby traffic, weather, and special use airspace. This capability can be valuable when evaluating an ATC-offered "direct" (i.e., shortcut) or when the pilot is considering making such a request.

An example of the utility of improved situation awareness occurred on the same San Antonio to Seattle flight of the "Cumulative Small Gains" discussion earlier. The example is in two parts: a "Plan Validation" benefit leading ultimately to an unexpected additional benefit. While proceeding to the EDLES intersection (see Figure 10), the pilot switched to TAP's manual mode and entered a route modification to fly from present position directly to the Yakima (YKM) navigational aid, effectively bypassing EDLES with a "shortcut." TAP predicted the maneuver would increase both fuel burn and flight time, an expected result given that TAP had earlier recommended EDLES as a more wind- 
efficient routing. This information reaffirmed the pilot's decision to remain on course toward EDLES (i.e., plan validation, though not the flight plan in this case). More generally, it provides an example wherein a common pilot or ATC action to improve flight cost (i.e., pursuing shortcuts) can sometimes be detrimental to flight cost, whereas using TAP can help avoid such unintended consequences.

Unexpectedly in this example, however, TAP's probe of the maneuver also indicated conflicting traffic crossing approximately 90 $\mathrm{nm}$ ahead at the ownship's altitude, FL340. Shortly thereafter and not unexpectedly given TAP's preview, ATC instructed the TAP aircraft to descend to FL320 for traffic. Retaining this new cruise altitude for the duration of the flight would have cost over $400 \mathrm{lbs}$ of fuel, according to TAP's prediction. Upon reaching the lower altitude, the pilot entered FL340 in manual mode (see Figure 11) and as expected, TAP again predicted the conflict. As soon as the conflict indication disappeared, the pilot requested a climb back to FL340 (immediately approved by ATC), thereby minimizing flight time at the less efficient altitude. As many pilots have experienced, ATC does not always offer an expeditious return from a conflict resolution maneuver. By providing supplemental information to the pilot (i.e., proximity of traffic to a proposed route modification), the use of TAP increased the pilots situation awareness of the surrounding airspace and thereby enabled the pilot to take proactive steps to restore flight efficiency at the earliest opportunity through a well-timed request to ATC.

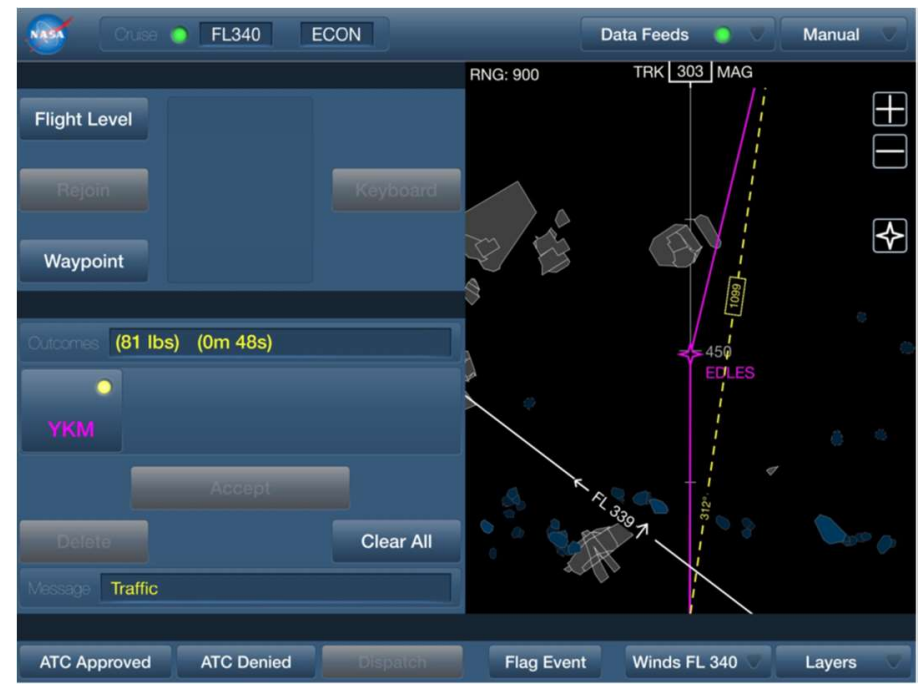

Figure 10. Example of improved situation awareness showing a cost-detrimental shortcut and crossing traffic.

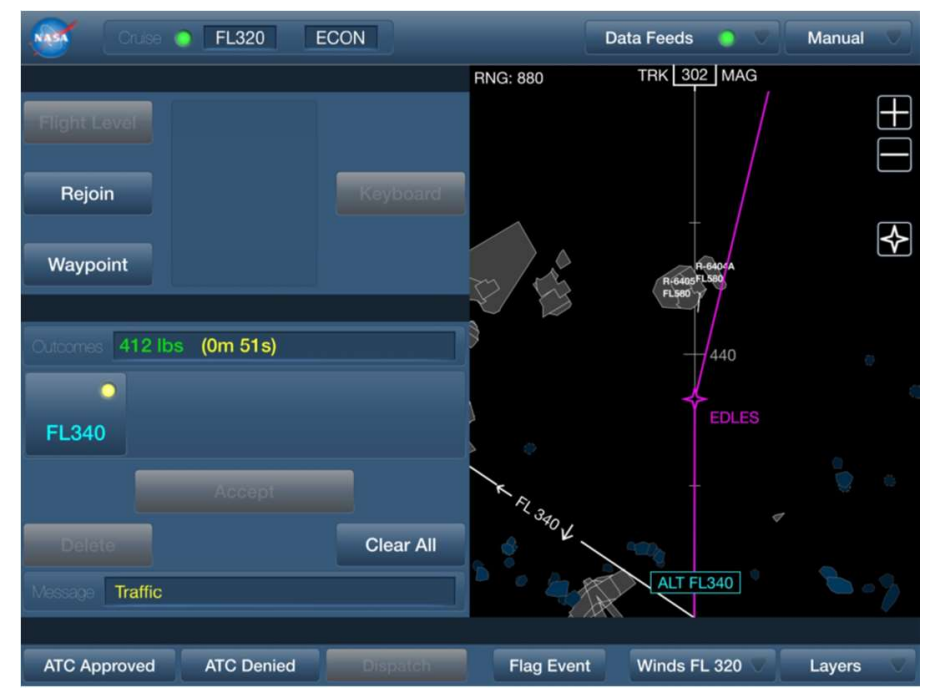

Figure 11. Monitoring TAP's conflict predictions of a proposed maneuver (as shown, climb from FL320 to FL340).

\section{Conclusion}

NASA and Alaska Airlines conducted an operational evaluation of Traffic Aware Strategic Aircrew Requests (TASAR), a NASA concept for cockpit-based, in-flight route optimization. The evaluation exercised NASA's prototype Traffic Aware Planner (TAP) software application for the "connected" Electronic Flight Bag (EFB) on three Boeing 737-900ER aircraft. Collaborating with NASA and Alaska on implementation of the TASAR system on the three aircraft were industry leaders Collins Aerospace, Gogo Commercial Aviation, and Aviation Communications \& Surveillance Systems (ACSS). The evaluation took place on 119 revenue-service flights in the period July 2018 to April 2019, 84 of which were determined to be valid for data analysis. These counts and the benefit results below are preliminary findings, based on the subset of data available at publication.

Of the 84 valid flights, $64 \%$ had at least one TAP-inspired, ATC-approved route modification. Across all valid flights including those with no requests or achieved benefits, the mean achieved benefit based on fuel burn and flight time savings was about $\$ 92$ per flight with a margin of error of about $\$ 59$ at $95 \%$ confidence level. Savings per flight ranged from $-\$ 381$ to $+\$ 1145$. Negative savings represented unrelated route changes that offset TAP gains. A number 
of factors were identified indicating the mean per-flight benefit measured in this evaluation may be conservative relative to the benefits likely to be achieved in regular airline operations.

Several TASAR benefit categories were observed in the operational evaluation: "Home Runs" that produced isolated cases of significant savings; "Cumulative Small Gains" that accumulated savings from several fine-tuning opportunities; and "Plan Validation" events where TAP confirmed the correctness of the flight plan with updated data, inhibiting unintended cost-inducing maneuvers. In addition, TAP provided the flight crew with additional information (e.g., nearby traffic affecting a proposed route modification) that increased their situation awareness of relevant airspace information and assisted pilots in proactive decision-making and effective ATC communications.

The operational evaluation has confirmed that TASAR is a viable and beneficial concept for airline operations, even with prototype technology. Opportunities for technology enhancement including incorporating new data sources (e.g. turbulence, onboard weather radar), implementing four-dimensional (4D) constraints and corresponding speed degree-of-freedom solutions, and adapting the technology for Flight Management System integration and data communications with ATC. The technology may also be adapted for non-airline operations, such as general aviation, business aviation, and military aircraft operations.

The TASAR concept embodies and authenticates the vision of the "connected" cockpit where onboard algorithms transform widely disparate data from onboard and off-board sources into operational savings. If adopted broadly, TASAR is also expected to initiate a culture change in which pilots engage more frequently and proactively in route optimization throughout the flight, elevating them to a role of an active trajectory manager in the dynamic airspace and propelling them on a path towards increased operational autonomy.

\section{References}

[1] Gogo, From the Ground Up: How the Internet of Things will Give Rise to Connected Aviation, Chicago: Gogo, LLC, 2016.

[2] M. G. Ballin and D. J. Wing, "Traffic Aware Strategic Aircrew Requests," in AIAA-2012-5623, 12th AIAA Aviation Technology, Integration, and Operations Conference, Indianapolis, 2012.

[3] S. E. Woods, R. A. Vivona, J. Henderson, D. J. Wing and K. E. Burke, "Traffic Aware Planner for Cockpit-based Trajectory Optimization," in AIAA-2016-4067, 16th AIAA Aviation Technology, Integration, and Operations Conference, Washington DC, 2016.

[4] D. J. Wing, M. G. Ballin, S. Koczo and R. A. Vivona, "Developing an On-Board Traffic-Aware Flight Optimization Capability for Near-Term Low-Cost Implementation," in AIAA-2013-4231, 13th AIAA Aviation Technology, Integration, and Operations Conference, Los Angeles, 2013.

[5] D. J. Wing, "Achieving TASAR Operational Readiness," in AIAA-2015-3400, 15th AIAA Aviation Technology, Integration, and Operations Conference, Dallas, 2015.

[6] J. Henderson, H. Idris and D. J. Wing, "Preliminary Benefits Assessment of Traffic Aware Strategic Aircrew Requests (TASAR)," in AIAA-2012-5684, 12th AIAA Aircraft Technoogy, Integration, and Operations Conference, Indianapolis, 2012.

[7] J. Henderson, "Annualized TASAR Benefit Estimate for Virgin American Operations," NASA/CR-2015-218786, 2015.

[8] J. Henderson, "Annualized TASAR Benefit Estimate for Alaska Airlines Operations," NASA/CR-2015-218787, 2015.

[9] S. Koczo, "Analysis of Operational Hazards and Safety Requirements for Traffic Aware Strategic Aircrew Requests (TASAR)," NASA/CR-2013-218002, 2013.

[10] S. Koczo, "TASAR Certification and Operational Approval Requirements - Analyses and Results," NASA/CR-2015-218708, 2015.

[11] D. A. Roscoe, R. A. Vivona, S. E. Woods, D. A. Karr and D. J. Wing, "Deploying a Route Optimization EFB Application for Commercial Airline Operational Trials," in Digital Avionics Systems Conference, Sacramento, CA, 2016.

[12] J. M. Maris, M. A. Haynes, D. J. Wing, K. A. Burke, J. Henderson and S. E. Woods, "Traffic Aware Planner (TAP) Flight Evaluation," in AIAA-2014-2166, 14th AIAA Aircraft Technology, Integration, and Operations Conference, Atlanta, 2014.

[13] D. J. Wing, K. A. Burke, J. Henderson, R. Vivona and J. Woodward, "Initial Implementation and Operational Use of TASAR in Alaska Airlines Flight Operations," in AIAA-2018-3043, 18th AIAA Aviation Technology, Integration, and Operations Conference, Atlanta, 2018.

[14] J. Henderson, D. J. Wing, K. Ballard and W. Cotton, "Alaska Airlines TASAR Operational Trial Achieved Benefits," NASA Technical Memorandum, expected 2019. 